\title{
Superheating systematics of crystalline solids
}

\author{
Sheng-Nian Luo a) and Thomas J. Ahrens \\ Lindhurst Laboratory of Experimental Geophysics, Seismological Laboratory, California Institute \\ of Technology, Pasadena, California 91125
}

(Received 1 October 2002; accepted 30 January 2003)

\begin{abstract}
Systematics of superheating $\left(\theta=T / T_{m}-1\right)$ of crystalline solids as a function of heating rate $(Q)$ are established as $\beta=A(Q)(\theta+1) \theta^{2}$, where the normalized energy barrier for homogeneous nucleation is $\beta=16 \pi \gamma_{\mathrm{ss}}^{3} /\left(3 k T_{m} \Delta H_{m}^{2}\right), T$ is temperature, $T_{m}$ melting temperature, $A$ a $Q$-dependent parameter, $\gamma_{\mathrm{sl}}$ interfacial energy, $\Delta H_{m}$ heat of fusion, and $k$ Boltzmann's constant. For all elements and compounds investigated, $\beta$ varies between 0.2 and 8.2. At 1 and $10^{12} \mathrm{~K} / \mathrm{s}, A=60$ and $31, \theta$ $=0.05-0.35$ and $0.06-0.45$, respectively. Significant superheating is achievable via ultrafast heating. We demonstrate that the degree of superheating achieved in shock-wave loading and intense laser irradiation as well as in molecular dynamics simulations $\left(Q \sim 10^{12} \mathrm{~K} / \mathrm{s}\right)$ agrees with the $\theta-\beta-Q$ systematics. (C) 2003 American Institute of Physics. [DOI: 10.1063/1.1563046]
\end{abstract}

Superheating $\left(\theta=T / T_{m}-1\right)$ of a crystalline solid occurs when the long-range order of the crystalline structure is maintained up to certain temperature $T$ above the equilibrium melting temperature $T_{m}$. Previously, the details of crystal melting and the temperature range over which solids may be superheated have been investigated. ${ }^{1-7}$ In contrast to undercooling of liquid prior to crystallization, experimental superheating of crystals is difficult as grain boundaries and free surfaces lower the energy barriers for melt nucleation. ${ }^{8,9}$ Special experimental designs ${ }^{5}$ and rapid heating are required to superheat crystalline solids. Catastrophic melting ${ }^{1,2}$ and homogeneous nucleation ${ }^{3,4}$ theories have been utilized to define the limits of superheating, and a wide range of superheating $(\theta \sim 0.1-2.0)$ is predicted. Here, we will investigate the systematics of nucleation energy barrier for elements and compounds, and the corresponding superheating as a function of heating rate. We also compare theory to superheating achieved in experiments and simulations.

Homogeneous nucleation of melt may be described via classical theories. ${ }^{9-14}$ Let $I$ be the rate per unit volume of steady-state homogeneous nucleation of melt in solid: ${ }^{14,15}$

$$
I=I_{0} \exp \left(-\frac{\Delta G_{c}}{k T}\right),
$$

where $\Delta G_{c}$ is the critical Gibbs free energy for nucleation, and $k$ is Boltzmann's constant. The prefactor $I_{0}$ can be obtained experimentally or theoretically. Consider a spherical liquid nucleus of critical radius within a crystal lattice, the critical nucleation energy ${ }^{14,15} \Delta G_{c}=16 \pi \gamma_{\mathrm{sl}}^{3} /\left(3 \Delta G_{\mathrm{sl}}^{2}\right)$ where $\gamma_{\mathrm{sl}}$ is the solid-liquid interfacial energy, and $\Delta G_{\mathrm{sl}}$ is the Gibbs free energy difference per unit volume between solid and liquid state, approximated as $\Delta H_{m}\left(T-T_{m}\right) / T_{m}$ where $\Delta H_{m}$ is the heat of fusion (assuming heat capacities of liquid and solid are approximately equal ${ }^{16}$ ). If we define the normalized energy barrier for nucleation $\beta$ as

$$
\beta\left(\gamma_{\mathrm{sl}}, \Delta H_{m}, T_{m}\right)=\frac{16 \pi \gamma_{\mathrm{sl}}^{3}}{3 \Delta H_{m}^{2} k T_{m}},
$$

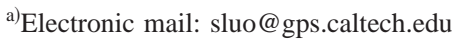

then $I$ can be written as $I=I_{0} f(\beta, \theta)$, with

$$
f(\beta, \theta)=\exp \left[-\frac{\beta}{(\theta+1) \theta^{2}}\right] .
$$

Nucleation rate $I$ is controlled by $f(\beta, \theta)$, essentially by $\beta$ at a given temperature. Equations (1)-(3) are also applicable to the undercooling case.

To estimate the magnitude of $\beta$, we note that $\gamma_{\mathrm{sl}}$ $\sim 0.1 \mathrm{~J} / \mathrm{m}^{2}, \quad T_{m} \sim 10^{3} \mathrm{~K}$ and $\Delta H_{m} \sim 10^{9} \mathrm{~J} / \mathrm{m}^{3}$, yields $\beta$ $\sim$ 1.2. Based on previous data, ${ }^{14} \beta$ for elements is calculated (Fig. 1). For Group IVB-IIB elements, $\beta$ is $0.9-3.1$, except for $\mathrm{Hg}$ (6.3). For most transition metals, $\beta \sim 1.8$. Due to the unproportionally lower $\Delta H_{m}$ and $T_{m}$, Group IIIA-VIA elements have larger $\beta$ values (2.5-8.2) except $\mathrm{Al}(1.5)$ and $\mathrm{Se}$ (0.2). Figure 1 demonstrates the periodic nature of $\beta$ for elements due to their periodic variations in electronic structure, with peaks occurring mostly at Group IIIA-VIA elements and $\mathrm{Hg}$. $\beta$ for compounds such as some alkali halides and silicates is similar. In general, $\gamma_{\mathrm{sl}}$ increases with $T_{m}$ and $\Delta H_{m}$, because $\gamma_{\mathrm{sl}}, T_{m}$, and $\Delta H_{m}$ are fundamentally related to binding energy. Thus, although $\beta$ is sensitive to $\gamma_{\mathrm{sl}}$, varia-

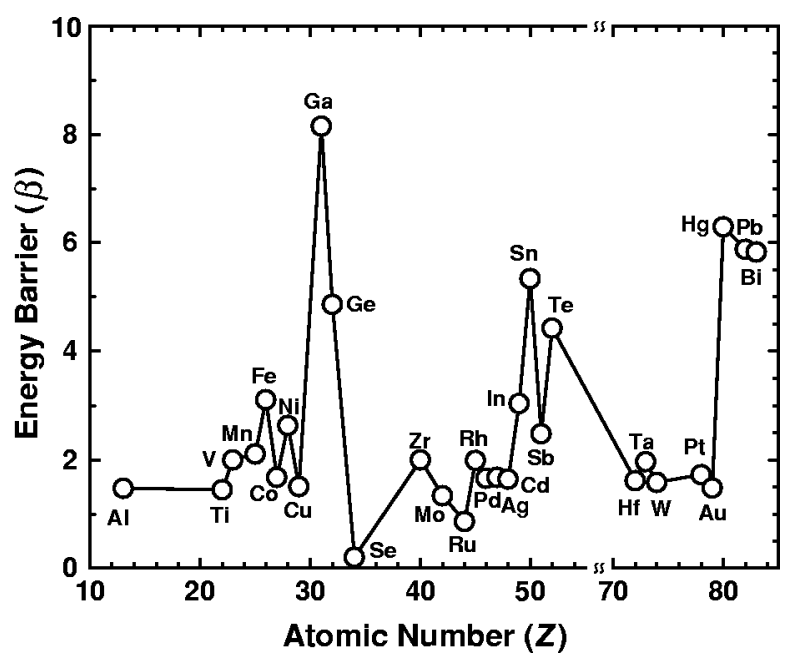

FIG. 1. Normalized energy barrier $\beta$ as a function of atomic number $Z$. Values for $\mathrm{V}, \mathrm{Mo}$, and $\mathrm{W}$ are estimated. 
tion in $\beta$ induced by that in $\gamma_{\mathrm{sl}}$ could be offset by those in $T_{m}$ and $\Delta H_{m}$. We also note that $\gamma_{\mathrm{sl}} \propto \Delta H_{m} / a^{2}$, where $a^{2}$ is the effective atomic surface area. ${ }^{12}$ Since $\beta$ lies between 0.2 and 8.2 for materials with a wide range of binding energy and other physical properties, we expect that $\beta$ remains at the same order under high pressures, (e.g., shock compression).

Given the $\beta$ systematics, next we will develop a $Q$-dependent scheme to probe the systematics of superheating characteristic of elements and compounds. The undercooling experiments on various elements and compounds were documented, ${ }^{14}$ and serve as the basis for our calculating the superheating at various heating rates. The parameters for undercooling such as $\gamma_{\mathrm{sl}}, \Delta H_{m}, T_{m}$, and molar volume $V$ can be regarded as equal to those for superheating. In the case of superheating, the probability ${ }^{14} x$ of $v$ moles parent phase containing no new phase (liquid) under heating rate $Q$ is

$$
x=\exp \left[-\frac{v T_{m} I_{0} V}{Q^{+}} \int_{1}^{\left(1+\theta^{+}\right)} f(\theta, \beta) d \theta\right],
$$

where + denotes superheating, and the expression is similar for undercooling case (denoted with - ). If we assume $I_{0}, v$, and $x$ for superheating are similar to those for undercooling, superheating $\theta^{+}$can be estimated from undercooling $\theta^{-}$ with Eq. (4). Given undercooling results ${ }^{14}$ under typical cooling rate $Q^{-}=1 \mathrm{~K} / \mathrm{s}$, superheating $\left(\theta^{+}\right)$under certain heating rate $Q^{+}$can be obtained by solving $\left(1 / Q^{-}\right) \int_{\left(1-\theta^{-}\right)}^{1} f(\theta, \beta) d \theta=\left(1 / Q^{+}\right) \int_{1}^{\left(1+\theta^{+}\right)} f(\theta, \beta) d \theta$. For superheating, we will consider two extreme cases: $Q^{+}$ $=1 \mathrm{~K} / \mathrm{s}$ (corresponding to $\theta_{s}^{+}$) and $10^{12} \mathrm{~K} / \mathrm{s}\left(\theta_{\mathrm{ns}}^{+}\right)$. These heating rates should be regarded as typical but not exact, because a factor of $10^{2}$ difference in $Q$ would yield a negligible difference in $\theta$ given a reasonable value of $f(\beta, \theta)$. Superheating at these heating rates is calculated in Fig. 2 for elements. Group IIIA-VIA elements (except $\mathrm{Se}$ ) can be superheated more than transition elements (except $\mathrm{Hg}$ ). The numerical relationship between $\beta$ and $\theta$ can be fitted with a simple analytical form

$$
\beta=A(Q)(1+\theta) \theta^{2},
$$

where $A$ is a $Q$-dependent parameter. $A=60$ and 31 at $Q$ $=1$ and $10^{12} \mathrm{~K} / \mathrm{s}$ respectively, and decreases with increasing $Q$. As a preliminary result, $A(Q)$ is fitted as $A(Q)$ $=60-2.4 \log Q$. While its exact physical meaning is not clear, $A$ could be regarded as defining a relative characteristic time scale for nucleation at various heating rates. For silicates and alkali halides, the degree of superheating agrees with the fits for elements. Superheating $\theta$ is inherently limited by material property $\beta$, and increases monotonically with $\beta$ and $Q$ (Fig. 2). Values of $\beta=0.2-8.2$ correspond to superheating of $0.05-0.35$ at $1 \mathrm{~K} / \mathrm{s}$ and $0.06-0.45$ at $10^{12} \mathrm{~K} / \mathrm{s}$, respectively. Crystalline solids can be superheated by $0.1 T_{m}-0.5 T_{m}$ regardless of heating rates, and for transition metals, $\theta \sim 0.1-0.3$. At similar heating and cooling rates, undercooling $\left(\theta^{-}\right)$is larger than superheating $\left(\theta^{+}\right)$. Despite significant differences in physical properties among these elements and compounds, superheating can be described by Eq. (5), which we expect to be valid for crystalline solids in general. Thus, we established the $\theta-\beta-Q$ sys-

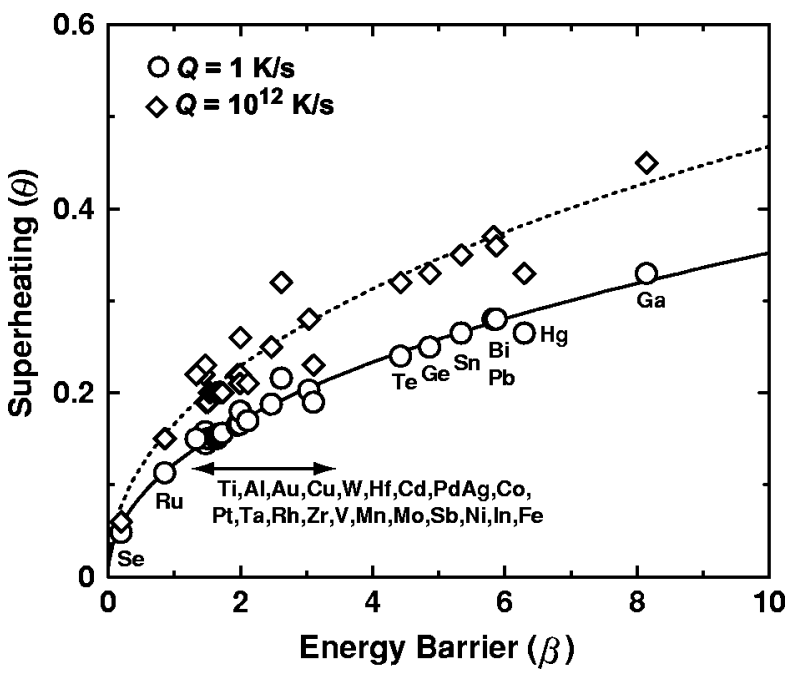

FIG. 2. Superheating vs normalized energy barrier for elements. Solid curve is the analytical fitting of $\theta-\beta$ pairs at $Q=1 \mathrm{~K} / \mathrm{s}$ (circles) to Eq. (5) with $A=60$. Dashed curve corresponds to $Q=10^{12} \mathrm{~K} / \mathrm{s}$ with $A=31$. Superheating of $\mathrm{Ni}$ is possibly overestimated (see Ref. 14).

tematics [Eq. (5) and Fig. 2] based on undercooling experiments and homogeneous nucleation theory.

During shock-wave loading via planar impact or intense laser irradiation, a solid is subjected to ultrafast heating. It is well known that pronounced superheating may occur in molecular dynamics (MD) simulations of perfect crystals with three-dimensional periodic boundaries. ${ }^{17}$ Melt nucleation during such processes can be regarded as homogeneous. In contrast to low heating rate experiments at $1 \mathrm{~K} / \mathrm{s}$ order, in shock-wave loading (e.g., planar impact), the shock-front rise time is on the order of $1 \mathrm{~ns}$, and temperature increase upon shock compression is on the order of $10^{3}-10^{4} \mathrm{~K}$; that is, $Q \sim 10^{12} \mathrm{~K} / \mathrm{s}$. $Q$ is similar in intense laser irradiation, depending on energy deposited, irradiation time, and material properties. Similar values of $Q$ apply for MD simulations. Melting under ultrafast heating has been investigated experimentally employing both planar impact and intense laser irradiation. Sound speed and temperature measurement at shock state unequivocally demonstrated superheating of metals, alkali halides, and silicates. ${ }^{6,18-25}$ Time-resolved electron diffraction and mass spectroscopy demonstrated the occurrence of superheating during intense laser irradiation. ${ }^{7,26,27}$ In Table I, we list the superheating results from planar impact and laser irradiation experiments. For planar impact, if $T_{H}^{1}$ is the lowest temperature on liquid Hugoniot and $T_{H}^{2}$ the highest temperature of superheated solid at corresponding shock pressure $P_{H}$, then superheating on Hugoniot $\theta_{H}^{+}$is $\left(T_{H}^{2}\right.$ $\left.-T_{H}^{1}\right) / T_{H}^{1}$. The comparison of $\theta_{H}^{+}$with $\theta_{\mathrm{ns}}^{+}$shows that $\theta_{H}^{+}$ (except for $\mathrm{Mg}_{2} \mathrm{SiO}_{4}$ ) is close to $\theta_{\mathrm{ns}}^{+}$, the prediction of superheating systematics, given the modest variation of energy barrier $\beta$ with pressure under shock loading and uncertainties in experiments. Similar results have been obtained for intense laser irradiation. Thus, the ultrafast heating experiments agree with the proposed systematics of superheating within experimental error, and possible $\beta$ variations due to pressure and phase changes. Similarly, MD simulations for bulk $\mathrm{Al}^{28}$ yielded $\theta=0.22$ (compared to $\theta_{\mathrm{ns}}^{+}=0.20$ ), and for $\mathrm{SiO}_{2}$ (e.g., at $\left.120 \mathrm{GPa}\right)^{29} \theta=0.33$ in accord with the system-

atics. 
TABLE I. Superheating by planar impact and laser irradiation. Numbers in bold fonts are estimated. Note that materials may be subjected to phase changes and decomposition at high pressures. The reference(s) for each material are in brackets.

\begin{tabular}{|c|c|c|c|c|c|}
\hline $\begin{array}{l}\text { Starting } \\
\text { material }\end{array}$ & $\begin{array}{c}P_{H} \\
(\mathrm{GPa})\end{array}$ & $\begin{array}{l}T_{H}^{1} \\
(\mathrm{~K})\end{array}$ & $\begin{array}{l}T_{H}^{2} \\
(\mathrm{~K})\end{array}$ & $\theta_{H}^{+}$ & $\theta_{\mathrm{ns}}^{+}$ \\
\hline \multicolumn{6}{|c|}{ Planar shock-wave impact } \\
\hline $\mathrm{Fe}[19,22]$ & 265 & 6000 & 7500 & 0.25 & 0.23 \\
\hline $\mathrm{V}[24]$ & 210 & 6000 & 7800 & 0.30 & 0.26 \\
\hline Mo $[20,25]$ & 385 & 9000 & 12000 & 0.33 & 0.19 \\
\hline $\mathrm{Ta}[18]$ & 295 & 7000 & 9000 & 0.29 & 0.23 \\
\hline $\mathrm{W}$ [25] & 410 & 10000 & 13000 & 0.30 & 0.20 \\
\hline $\mathrm{CsBr}[21]$ & 38 & 4000 & 5000 & 0.25 & 0.22 \\
\hline $\mathrm{KBr}[21]$ & 28 & 3500 & 4200 & 0.20 & 0.21 \\
\hline Fused quartz [6] & 70 & 4500 & 5300 & 0.18 & $\ldots$ \\
\hline Quartz [6] & 113 & 4800 & 6100 & 0.27 & $0.34^{\mathrm{a}}$ \\
\hline $\mathrm{Mg}_{2} \mathrm{SiO}_{4}[23]$ & 130 & 4300 & 7000 & 0.63 & $\cdots$ \\
\hline \multicolumn{6}{|c|}{ Laser irradiation } \\
\hline $\mathrm{Al}[26]$ & 0 & 933 & $1300^{\mathrm{b}}$ & 0.39 & 0.20 \\
\hline $\mathrm{Pb}[27]$ & 0 & 601 & 721 & 0.20 & 0.37 \\
\hline GaAs [7] & 0 & 1511 & 2061 & 0.36 & $\ldots$ \\
\hline
\end{tabular}

${ }^{\mathrm{a}}$ Value is for quartz at ambient pressure.

${ }^{b}$ Value for irradiation flux of $7 \mathrm{~mJ} / \mathrm{cm}^{2}$ is adopted.

In the previous discussion, we assumed $I_{0}$ is similar for undercooling and superheating cases. $I_{0}$ for undercooling could differ by several orders from that for superheating. However, due to the nature of exponential function $f(\theta, \beta)$, the superheating estimated this way should not deviate much from the true value as demonstrated by experiments and simulations. The observed superheating systematics manifest that catastrophic nucleation is limited by $\beta$ and $Q$. The superheating systematics are fundamentally attributed to atomic forces (e.g., binding energy) and characteristic of crystalline solids, while this phenomenological relationship needs to be established from first principles. With the $\theta-\beta-Q$ systematics, we can predict superheating at certain $Q$ if $\beta$ (essentially, $\gamma_{\mathrm{sl}}$ ) is known, or vice versa. Investigation of the parameter $\gamma_{\mathrm{sl}}$ with experimental and theoretical (e.g., MD) techniques under high pressure and temperatures, remains challenging. Despite these uncertainties, it is clear that crystals can be superheated under ultrafast conditions, and the degree of superheating prior to thermal melting is in the range of $0.1 T_{m}$ to $0.5 T_{m}$. While the predicted superheating from $0.05 T_{m}$ to $0.35 T_{m}$ at $1 \mathrm{~K} / \mathrm{s}$ is seldom observed because free-surface effects dominate at low heating rates, the super- heating systematics will be of practical importance for fast dynamic loading as well as molecular dynamics simulation of melting.

This work has been supported by NSF Grant EAR0207934. Discussions with Z. Gong, D. Stevenson, D. Swift, and R. Jeanloz are appreciated. Contribution No. 8878, Division of Geological and Planetary Sciences, California Institute of Technolgy.

${ }^{1}$ H. J. Fecht and W. L. Johnson, Nature (London) 334, 50 (1983).

${ }^{2}$ J. L. Tallon, Nature (London) 342, 658 (1989).

${ }^{3}$ K. Lu and Y. Li, Phys. Rev. Lett. 80, 4474 (1998).

${ }^{4}$ B. Rethfeld, K. Sokolowski-Tinten, D. von der Linde, and S. I. Anisimow, Phys. Rev. B 65, 092103 (2002).

${ }^{5}$ D. R. Uhlmann, J. Non-Cryst. Solids 41, 347 (1980).

${ }^{6}$ G. A. Lyzenga, T. J. Ahrens, and A. C. Mitchell, J. Geophys. Res., [Solid Earth] 88, 2431 (1983).

${ }^{7}$ N. Fabricius, P. Hermers, and D. von der Linde, Solid State Commun. 58, 239 (1986).

${ }^{8}$ J. Frenkel, Kinetic Theory of Liquids (Oxford University Press, New York, 1946).

${ }^{9}$ D. Turnbull, Solid State Phys. 3, 225 (1956).

${ }^{10}$ D. Turnbull and J. C. Fisher, J. Chem. Phys. 17, 71 (1949).

${ }^{11} \mathrm{~J}$. W. Christian, The Theory of Transformation in Metals and Alloys (Pergamon, New York, 1965).

${ }^{12}$ A. G. Walton, in Nucleation edited by A.C. Zettlemoyer (Marcel Dekker, New York, 1969), p. 225.

${ }^{13}$ V. I. Motorin and S. L. Musher, J. Chem. Phys. 81, 465 (1984).

${ }^{14}$ K. F. Kelton, Solid State Phys. 45, 75 (1991).

${ }^{15}$ D. Porter and K. E. Easterling, Phase Transformations in Metals and Alloys (Wokingham, Berkshire, 1981).

${ }^{16}$ D. Turnbull, J. Chem. Phys. 20, 411 (1952).

${ }^{17}$ M. P. Allen and D. Tildesley, Computer Simulation of Liquids (Clarendon, Oxford, 1987)

${ }^{18}$ J. M. Brown and J. W. Shaner, Shock Waves in Condensed Matter, edited by J. R. Asay, R. A. Graham, and G. K. Straub (Elsevier Science, New York 1983).

${ }^{19}$ J. M. Brown and R. G. McQueen, J. Geophys. Res., [Solid Earth] 91, 7485 (1986).

${ }^{20}$ R. S. Hixson, D. A. Boness, J. W. Shaner, and J. A. Moriarty, Phys. Rev. Lett. 62, 637 (1989).

${ }^{21}$ D. A. Boness and J. M. Brown, Phys. Rev. Lett. 71, 2931 (1993).

${ }^{22}$ C. Y. Yoo, N. C. Holmes, M. Ross, D. J. Webb, and C. Pike, Phys. Rev. Lett. 70, 3931 (1993).

${ }^{23}$ K. G. Holland and T. J. Ahrens, Science 275, 1623 (1997).

${ }^{24}$ C. Dai, X. Jin, X. Zhou, J. Liu, and J. Hu, J. Phys. D 34, 3064 (2001).

${ }^{25}$ R. S. Hixson and J. N. Fritz, J. Appl. Phys. 71, 1721 (1992).

${ }^{26}$ S. Williamson, G. Mourou, and J. C. M. Li, Phys. Rev. Lett. 52, 2364 (1984).

${ }^{27}$ J. W. Herman and H. E. Elsayed-Ali, Phys. Rev. Lett. 69, 1228 (1992).

${ }^{28}$ Z. H. Jin and K. Lu, Philos. Mag. Lett. 78, 29 (1998).

${ }^{29}$ S.-N. Luo, J. L. Mosenfelder, P. D. Asimow, and T. J. Ahrens, Phys. Usp. 40, 435 (2002). 